\title{
Analysis of Thermal Stress Intensity Factors for Double Cantilever Beams under Pulsed Laser
}

\author{
Zhongxiangchu ${ }^{1, a}$, xiaominzhang, songpeng, ziminyan \\ ${ }^{1}$ School of Chongqing University \\ azhongxiangchu@163.com
}

Keywords: photothermal microactuator, interlayer cracks, displacement extrapolation method, generalized heat conduction

Abstract: This study adopts a 3D model of a double cantilever beam with interlayer defects under
pulsed laser. The type II stress intensity factor $K_{\mathrm{II}}$ at the 3D crack tip of the interlayer and its
variation with time are obtained via displacement extrapolation under the classical thermoelasticity
and generalized thermoelasticity, respectively, and using the non-Fourier heat conduction equation.
The effects of thickness ratio, crack tip position, and heat source at different positions are discussed.

\section{Introduction}

A microactuator based on microscale photothermal expansion is the core component of a photothermal microactuator. Using the thermal expansion of materials under laser irradiation as the actuating force for micron-level movement, microactuators are usually shaped into double-cantilever beams. The reliability of microactuators under pulsed thermal shock is an important research topic pertaining to related instruments. One cause of the structural failure of microactuators is the stripping or peeling caused by interlayer cracks. Hence, basic reliability research focuses on the stress intensity factors that contribute to such interfacial cracks ${ }^{[1,2]}$. As a result of the dual-material model being designed in the micron dimension, in which microstructures similar to interlayer cracks may form, many scholars have introduced non-Fourier heat conduction theories to solve problems related to the thermal stress intensity factors of interlayers. B.L. Wang studied interfacial cracks under non-Fourier heat conduction theories and obtained analytic solutions for the stress intensity factor at the crack tip infinitely. In the aforementioned studies, idealized 2D cracks were discussed extensively. To further discuss 3D interfacial cracks, Ayhan et al. ${ }^{[3,4]}$ established the extended finite element method, by which stress intensity factors could be directly obtained in the analysis process. Arash Zamani et al. ${ }^{[5]}$ obtained the dynamic thermal stress intensity factors $K_{\mathrm{I}}$ and $K_{\mathrm{II}}$ of interlayer cracks under thermal shock and varying with time by establishing two corresponding control equations based on classical heat conduction and generalized heat conduction and using interactive integration. In the present study, a 3D model is established for dual-material interlayer cracks, and under the thermal shock of pulsed laser, thermal coupling control equations based on classical heat conduction and non-Fourier heat conduction are used as heat conduction modes. Then, the stress intensity factors of 3D interlayer cracks are studied using the displacement extrapolation method. The effects of model dimension, heat source position, and other elements on the thermal stress intensity factors are obtained. The stress intensity factors calculated in the two heat conduction modes are subsequently compared.

\section{Computational model and parameters}

In this study, we establish a 3D dual-material cantilever model with an upper layer made of $\mathrm{SiO}_{2}$ and a lower layer made of polymer SU-8. The material parameters are given in Table 1 . The geometry of the substrate of SU-8 is as follows: length $L_{1}=1000 \mu \mathrm{m}$, width $b_{1}=100 \mu \mathrm{m}$, and height $h_{1}=50 \mu \mathrm{m}$. For the upper $\mathrm{SiO}_{2}$ layer, its geometry is as follows: length $L_{2}=1000 \mu \mathrm{m}$, width $b_{2}=100 \mu \mathrm{m}$, and height $h_{2}=10 \mu \mathrm{m}$. 
Table 1 material parameter

\begin{tabular}{ccccccc}
\hline Material & $\rho\left(\mathrm{kg} / \mathrm{m}^{3}\right)$ & $C_{\varepsilon}(\mathrm{J} / \mathrm{kg} \cdot \mathrm{K})$ & $E(\mathrm{GPa})$ & $v$ & $\alpha(1 / \mathrm{K})$ & $k(\mathrm{~W} / \mathrm{m} \cdot \mathrm{K})$ \\
\hline $\mathrm{SU}-8$ & 1190 & 1200 & 1.7 & 0.3 & $5 \times 10^{-5}$ & 0.2 \\
$\mathrm{SiO}_{2}$ & 2200 & 730 & 71 & 0.16 & $6 \times 10^{-7}$ & 1.4 \\
\hline
\end{tabular}

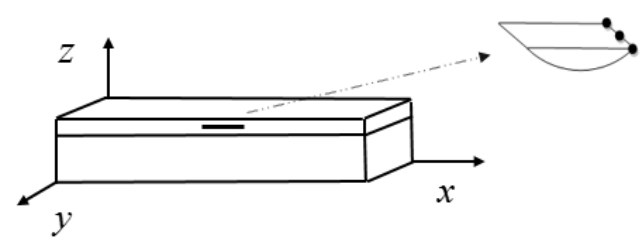

Fig. 1 3D dual-material cantilever model with interlayer defects

The geometry of the interlayer cracks is as follows: $10 \mu \mathrm{m}$ and $2 \mu \mathrm{m}$ along the $x$ and $y$-axes and $0.2 \mu \mathrm{m}$ along the $z$-axis. By averaging the stress intensity factors for five points at the crack tip along the $y$-axis, the final stress intensity factor $K$ is obtained. The boundary conditions are set as follows: the displacements along the $x^{-}, y^{-}$, and $z$-axes on the left side of the dual-material cantilever and the rotation of planes $x-y$ and $y-z$ are restricted. A uniform and asymmetrical Gaussian laser pulse is applied on the upper surface of the dual-material cantilever, whose temporal profile is given below. $I(t)=I_{0} t / t_{p}^{2} \exp \left(-t / t_{p}\right)$. where $t_{p}$ denotes the pulse duration and $I_{0}$ denotes the pulse energy density $\left(\mathrm{J} / \mathrm{m}^{2}\right)$, which indicates the carried laser energy per unit area. In laser heating, laser, assumed as a circular heat source on a surface, is quantified into a mathematical model in the form of a boundary heat flux. The boundary conditions are set within the circular region $R$ with the center of the upper surface as the center and the radius as $r=30 \mu \mathrm{m}$.

$$
q(x, y, z, t)_{x \in R}=\left\{\begin{array}{cc}
I & 0 \leq t \leq t_{w} \\
0 & t>t_{w}
\end{array} \text { where } t_{w}=1 \mathrm{~s}, I_{0}=10^{5} \mathrm{~J} / \mathrm{m}^{2} \text {. Initial condition } t=0\right. \text {. The }
$$

displacement is zero, and the initial temperature is $300 \mathrm{~K}$.

Considering such a typical transient heat conduction problem in the micro scale and the heat conduction characterized by typical non-Fourier effects over a small time scale, we derive the generalized L-S-type thermal coupling control equation and present it as follows.

$$
\begin{gathered}
k T,_{j j}=\rho C_{\varepsilon} \dot{T}+\rho C_{\varepsilon} \tau \ddot{T}+T_{0} \beta\left(\dot{u}_{j},{ }_{j}+\tau \ddot{u}_{j},{ }_{j}\right) \\
(\lambda+G) u_{j},_{j k}+G \nabla^{2} u_{k}-\beta T,_{k}+f_{k}-\rho \ddot{u}_{k}=0
\end{gathered}
$$

where $u_{j}$ and $T$ respectively denote the stress tensor and temperature. In the present work, the relative reference temperature is $T_{0}=300 \mathrm{~K}$, and the equivalent body force is $f_{k}=0$. Moreover, $k$, $\rho$, and $C_{\varepsilon}$ respectively denote the conductive coefficient, density, and specific heat at constant strain. Assuming that $\beta$ is the temperature coefficient, the relations between the available lame constant $\lambda, G$, and the linear expansion coefficient $\alpha$ are presented below. $\beta=(3 \lambda+2 G) \alpha$. Compared with the thermal coupling control equation for classical heat conduction, the relaxation time $\tau$ of the L-S-type thermal coupling control equation is added. The presence of relaxation time $\tau$ indicates the heat transfer in the medium at a limited speed. When $\tau=0$, this is the classical thermal coupling control equation. Under thermal shock and in a small space-time scale, the heat conduction with a significantly refined structure or non-uniform inner structure exhibits 
distinct non-Fourier characteristics. In this work, we use the PDE module of COMSOL, as well as the thermal coupling equation and the generalized thermal coupling equation, to calculate the displacement field and stress field at the crack tip.

\section{Numerical computation and discussions}

Based on the newly defined stress intensity factor, the asymptotic solution for crack tip opening displacement (CTOD) can be expressed as ${ }^{[1]}$

$$
\begin{aligned}
\left(\delta_{2}+i \delta_{1}\right)_{r \rightarrow 0} & =\frac{c_{1}+c_{2}}{2 \sqrt{2 \pi}(1+2 i \varepsilon) \cosh (\varepsilon \pi)} K r^{\frac{1}{2}+i \varepsilon} \\
\left(\delta_{3}\right)_{r \rightarrow 0} & =\sqrt{\frac{2}{\pi}}\left(\frac{1}{\mu_{1}}+\frac{1}{\mu_{2}}\right) K_{\mathrm{III}} r^{\frac{1}{2}}
\end{aligned}
$$

Where, $c_{i}=\left(1+\kappa_{i}\right) / \mu_{i}$. Both the stress field and displacement field near the crack tip are calculated using finite element software. With the corresponding CTODs $\delta_{1}, \delta_{2}$, and $\delta_{3}$ obtained and substituted in the above equation, the stress intensity factor $K$ of the same series with different values of $r$ is obtained. On the basis of a linear extrapolation of these values, the stress intensity factor when $r=0$ is obtained.

Fig. 2 shows the changing trend of the center $\sigma_{z}$ of the upper surface of cracks with time. The stress at the center of the upper surface of the cracks along the $z$-axis for the duration is a negative value, and the cracks are constantly under pressure. In this study, the variation of the type II thermal stress intensity factor with time under pulsed laser is mainly studied. Figs. 3 and 4 respectively show the changing trends in the stress intensity factors on the left and right sides and the changing trends with different material thicknesses. Subtle differences can be observed between the left and right sides of the 3D cracks exhibiting mechanical behavior. Moreover, the variation in $K_{\mathrm{II}}$ of different substrate thicknesses with time exhibits a waveform similar to that of the applied heat flux. After a rapid increase with time, it is slowly reduced to 0 . As the substrate thickness increases, $K_{\mathrm{II}}$ also increases, and its peak value is significantly affected. The changing trend is similar to the variation in temperature. Fig. 5 shows the variation of $K_{\mathrm{II}}$ at the crack tip with different delays. When $\tau=10^{-5} \mathrm{~s}$, the changing trend of the $K_{\mathrm{II}}$ at the crack tip shows significant fluctuations. The simulated peak value of the $K_{\text {II }}$ at the crack tip under the generalized L-S-type thermoelastic theory is higher than those under classical thermoelastic theories.

\section{Summary}

From the study, we know that the the stress intensity factors on the left and right sides are different. The variation in $K_{\mathrm{II}}$ of different substrate thicknesses with time exhibits a waveform similar to that of the applied heat flux. As the substrate thickness increases, $K_{\mathrm{II}}$ also increases, and its peak value is significantly affected. The simulated peak value of the $K_{\mathrm{II}}$ at the crack tip under the generalized L-S-type thermoelastic theory is higher than those under classical thermoelastic theories. 

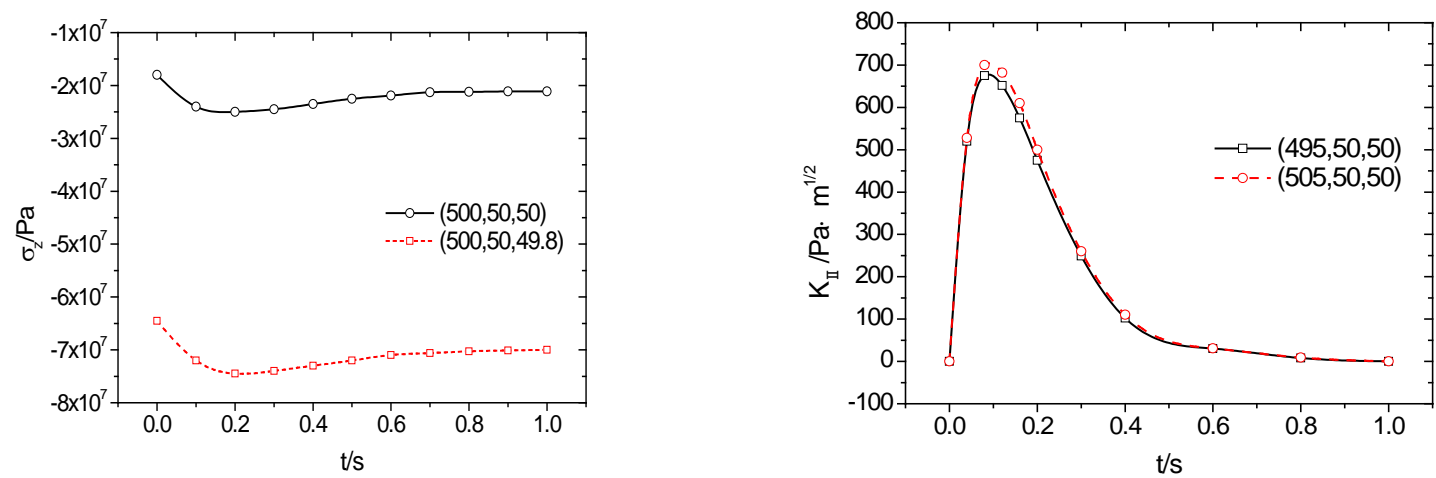

Fig. 2 center $\sigma_{z}$ change with time

Fig. 3 the changing trends of $K_{\mathrm{II}}$ on both left and right side of the crack

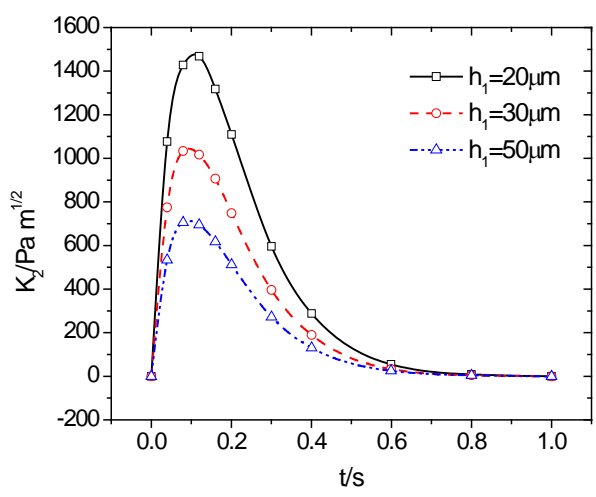

Fig. 4 the changing trends of $K_{\mathrm{II}}$ with thicknesses

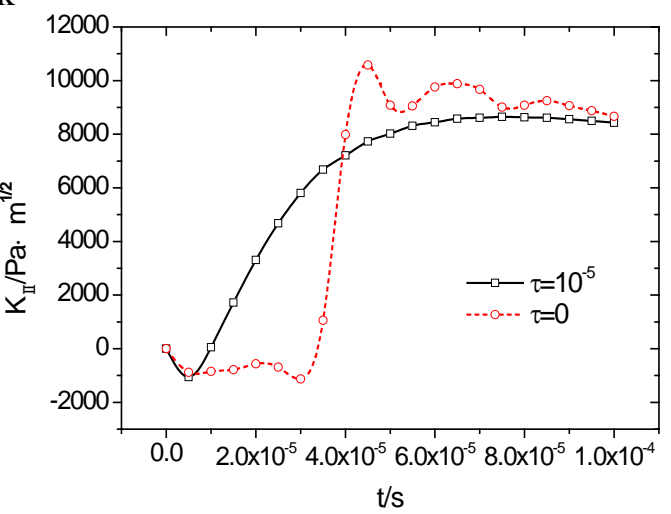

Fig. 5 the changing trends of $K_{\mathrm{II}}$ under different material different thermoelastic theories

\section{References}

[1] Hutchinson JW, Suo Z, Mixed mode cracking in layered materials[J]. Advances in applied mechanics, 1992,2(29):63-191

[2] MURAKAMI Y. Stress intensity factors handbook [M]. Oxford: Pengamon Press, 1987: 1100- 1105

[3] Ayhan SO, Nied HF, Stress intensity factors for three-dimensional surface cracks using enriched finite elements[J]. Int J Numer Methods Eng 2002, 54(6):899-921

[4] Ayhan AO, Kaya AC, Nied HF, Analysis of three-dimensional interface cracks using enriched finite elements[J]. Int J fract 2006, 143(3):255-276

[5] Arash Zamanni, Richard B, Hetnarski, M.Reza Eslami, Second sound in a cracked layer based on lord-shulman theory[J], Journal of thermal stress, 2011,34:181-200. 